Radial and Nonradial Pulsations as Probes of Stellar Physics

ASP Conference Series, Vol. 259, 2002

C. Aerts, T.R. Bedding, \& J. Christensen-Dalsgaard, eds.

\title{
Cycle to Cycle Irregularities in the Monoperiodic $\beta$ Cephei Star BW Vulpeculae
}

\author{
D. Garnier, N. Nardetto, P. Mathias
}

Observatoire de la Côte d'Azur, Nice, France

D. Gillet

Observatoire de Haute-Provence, St Michel l'Observatoire, France

\author{
A.B. Fokin \\ Institute for Astronomy, Academy of Sciences, Moscow, Russia
}

\begin{abstract}
High spectral and time resolution observations show that the monoperiodic $\beta$ Cephei star BW Vul presents differences in both line profiles and velocities from one cycle to another, which may be due to the consequence of the passage of two strong shock waves.
\end{abstract}

\section{High resolution observations}

The large amplitude $\beta$ Cephei star BW Vul (B2 III) is known to undergo two shock waves per pulsation period (Mathias et al., 1998):

- one is attributed to the now classical $\kappa$-mechanism from iron lines, and is responsible for the expansion motion. It is hereafter called the "outshock".

- the other, occurring during the end of the contraction motion, is due to the large velocity gradient between the top and the strongly decelerating bottom of the layer. It is hereafter called the "in-shock".

Between the in- and out-shocks, the stellar astmosphere is usually considered to be more or less at rest: this phase is called "stillstand".

In some other large amplitude stars where shock waves are present, such as RV Tauri (Gillet, 1992) and RR Lyrae (Chadid, 2000), it has been shown that non-linear motions could lead to irregularities in the radial velocity curves. Are such irregularities also present in the largest amplitude $\beta$ Cephei star?

Spectra were obtained at the Observatoire de Haute-Provence (France) with the $1.52 \mathrm{~m}$ telescope using the AURELIE spectrograph during 7 consecutive nights: from August 14 to 20, 2000. The spectral resolution was around 25000 along a $120 \AA$ spectral range centered on the Si III $4555 \AA$ line. The temporal resolution is very good: $2 \mathrm{~min}$, which corresponds to more than 100 spectra per pulsation period. The measured signal-to-noise ratio is between 100 and 150 . 


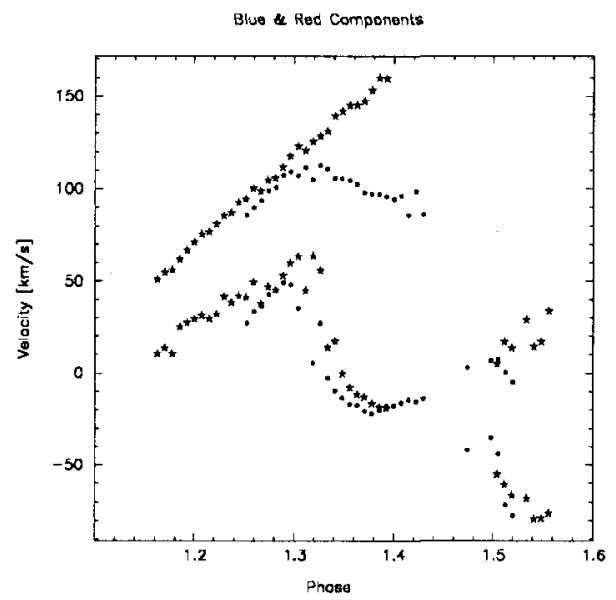

Figure 1. Comparison between the evolution of blue and red components during line doubling phases for nights August 15 (stars) and August 20 (dots) for the $\lambda \lambda 4553$ Si III line.

\section{Results}

The two strong shock waves induce two line doubling phenomena, one component being formed above the other. Therefore, we considered two distinct, Gaussian, components during line doubling phases. Fig. 1 shows the resulting comparison between two nights.

The slope associated to the red component of the first discontinuity is obviously different for the two nights. Since we are considering a ballistic motion, it implies that the effective gravity at a given phase is not the same for the two different cycles. We obtained $27.2 \mathrm{~m} \mathrm{~s}^{-2}$ for the night August 20 while the stellar gravity should be $22.6 \mathrm{~m} \mathrm{~s}^{-2}$. Hence, cycle-to-cycle differences are well present and occur mainly during the phases of shocks propagation. Among the 7 cycles we observed, half of them resemble that of night August 20, the other that of night August 15: no real intermediate case is noted. These differences are also present in the residual flux, FWHM and equivalent width variations. Also, the average value of the stillstand position can vary by about $10 \mathrm{~km} \mathrm{~s}^{-1}$, therefore it cannot be considered a phase of rest of the atmosphere. Because of the stillstand and equivalent width variations, it seems that the out-shock is mainly the origin of these changes.

\section{References}

Chadid, M. 2000, A\&A, 359, 991

Gillet, D. 1992, A\&A, 259, 215

Mathias, P., Gillet, D., Fokin, A.B., \& Cambon, T. 1998, A\&A, 339, 525 